\title{
TITLE:
}

\section{Glass transition of hard sphere systems: Molecular dynamics and density functional theory}

$\operatorname{AUTHOR}(\mathrm{S})$ :

Kim, K; Munakata, T

\section{CITATION:}

Kim, K ... [et al]. Glass transition of hard sphere systems: Molecular dynamics and density functional theory. Physical Review E 2003, 68(2): 021502.

ISSUE DATE:

2003-08

URL:

http://hdl.handle.net/2433/50290

RIGHT:

Copyright 2003 American Physical Society 
PHYSICAL REVIEW E 68, 021502 (2003)

\title{
Glass transition of hard sphere systems: Molecular dynamics and density functional theory
}

\author{
Kang Kim* and Toyonori Munakata ${ }^{\dagger}$ \\ Department of Applied Mathematics and Physics, Graduate School of Informatics, Kyoto University, Kyoto 606-8501, Japan
}

(Received 5 November 2002; published 12 August 2003)

\begin{abstract}
The glass transition of a hard sphere system is investigated within the framework of the density functional theory (DFT). Molecular dynamics (MD) simulations are performed to study the dynamical behavior of the system on the one hand and to provide the data to produce the density field for the DFT on the other hand. Energy landscape analysis based on the DFT shows that there appears a metastable (local) free energy minimum representing an amorphous state as the density is increased. This state turns out to become stable, compared with the uniform liquid, at some density around which we also observe a sharp slowing down of the $\alpha$ relaxation in the MD simulations.
\end{abstract}

DOI: 10.1103/PhysRevE.68.021502

PACS number(s): 64.70.Pf, 64.60.Cn, 61.20.Ja

Understanding the universal mechanism of the glass transition is one of the major challenges in current condensed matter physics. From a dynamical point of view, we would like to know how a drastic slowing down near the transition point (temperature or density) and an eventual exceeding of the relaxation time over the experimental time scale are realized [1,2]. Energetically or statically, it is asked whether a thermodynamic glassy state with an amorphous arrangement of particles has lower free energy than a liquid state of uniform density. In order to answer these questions, many efforts have been devoted to real experiments, computer simulations, and theories in the last few decades.

As one of the theories to study supercooled liquids and glasses, the density functional theory (DFT) is recently gathering much attention [3]. The DFT is now a conventional method to study the freezing [4,5] and other transitions. The glass transition has been investigated also based on the DFT by some workers $[6-10]$. In the earlier works [6-10], the random close packing (RCP) of hard spheres has been produced by Bennett's algorithm [11] and the free energy from the DFT with the input density field supplied by the RCP data has been calculated. Singh et al. [6] showed that the hard sphere glassy state becomes more stable than a uniform liquid at the critical density $n_{g} \sigma^{3}=1.14$, suggesting that there exists a kind of thermodynamic (later called a random first-order [12]) glass transition. Here, $\sigma$ is the hard sphere diameter and $n$ is the number density of the system. It is remarked here that since the RCP configurations were produced by a kind of aggregation method, we cannot study the dynamic aspects of the glass transition found by the energetics based on DFT.

The purpose of this paper is first to produce a supercooled and a glassy state for a one-component hard sphere system, relying on the uniform compression molecular dynamics (MD) method recently developed by Lubachevsky and Stillinger [13], and then to study both dynamic and static properties of the state. Especially from the particle configuration

\footnotetext{
*Present address: Department of Physics, Kyoto University, Kyoto 606-8502, Japan. Electronic address: kin@scphys.kyotou.ac.jp

${ }^{\dagger}$ Electronic address: munakata@amp.i.kyoto-u.ac.jp
}

data, we can discuss free energy within the DFT framework. This MD approach, in conjunction with the DFT, enables us to study both dynamic and static aspects of the transition in contrast with Bennett's approach.

Our system consists of $N=1372$ identical hard spheres with mass $m$ and diameter $\sigma$ in a cubic box of volume $V$ with periodic boundary conditions. Throughout this paper, the units of length and time are $\sigma$ and $\sqrt{m \sigma^{2} / k_{B} T}$, respectively, where $k_{B}$ is Boltzmann's constant and $T$ is the temperature [14]. It should be noted that the temperature is fixed as $k_{B} T=1$ in the course of the MD simulations.

To begin with, we briefly explain our MD method to obtain glassy states of a one-component hard sphere system. Employing the standard Alder and Wainwright algorithm $[15,16]$, we first generate the equilibrium liquid state at density $\tilde{n}=0.86$. It is well known that the fluid system freezes at $\tilde{n}_{f} \simeq 0.94$. To avoid crystallization and to obtain amorphous glassy states, Lubachevsky and Stillinger introduced a compressing (or quenching) procedure [13], in which they actually increased the diameter $\sigma$ with a constant rate of expansion during MD simulations. The dimensionless expanding rate $\Gamma$ is defined as

$$
\Gamma=\frac{d \sigma(t)}{d t} \sqrt{\frac{m}{k_{B} T}},
$$

and $\Gamma=0.01$ is chosen in our simulations. From the initial state $\tilde{n}=0.86$, we expanded each sphere with the rate $\Gamma$ and could obtain various high-density states $\tilde{n}=0.86,0.94,1.02$, $1.06,1.10,1.14,1.18$, and 1.21 , without crystallization.

Let us first study the static structure of the system. For this purpose, the radial distribution function $g(r)$, which is defined by

$$
g(r)=\frac{1}{n N}\left\langle\sum_{i \neq j}^{N} \delta\left(\boldsymbol{r}+\boldsymbol{r}_{i}-\boldsymbol{r}_{j}\right)\right\rangle,
$$

is calculated, where $\boldsymbol{r}_{\boldsymbol{i}}$ represents the positions of the $i$ th particle and $\langle\cdots\rangle$ denotes the ensemble average over different configurations. In Fig. 1, we plotted $g(r)$ for densities $\tilde{n}$ $=0.94,1.06,1.14$, and 1.21. It is noted that there is no sign of crystallization, which would be reflected in the sharp 


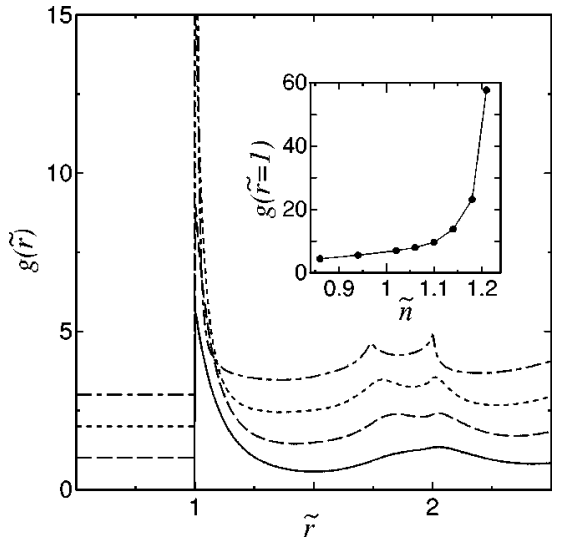

FIG. 1. The radial distribution function $g(\tilde{r})$ obtained for $\tilde{n}$ $=0.94$ (solid line), 1.06 (dashed line), 1.14 (short dashed line), and 1.21 (dot-dashed line). Inset: contact value of radial distribution function $g(\tilde{r}=1)$ as a function of $\tilde{n}$. The units of $\tilde{r}$ and $\tilde{n}$ are $\sigma$ $(\tilde{r}=r / \sigma)$ and $\sigma^{-3}\left(\tilde{n}=n \sigma^{3}\right)$, respectively.

peaks of $g(r)$ at some characteristic lattice spacings. Instead, $g(r)$ for higher density $(\tilde{n} \geqslant 1.14)$ shows splitting of the second peak, which is a familiar characteristic of a glassy state of a simple liquid. Furthermore, we notice that the contact value $g(\tilde{r}=1)$ shows an anomalous increase as the density increases (see the inset), which corresponds to the fact that the pressure increases drastically with increasing $\tilde{n}$. Incidentally, it should be remarked that the forms of $g(r)$ agree qualitatively with those illustrated in Ref. [11].

We next consider the dynamic aspects of the hard sphere glasses by calculating the incoherent intermediate scattering function $F_{s}(q, t)$, which is defined by

$$
F_{s}(q, t)=\left\langle\frac{1}{N} \sum_{j=1}^{N} \exp \left[i \boldsymbol{q} \cdot \Delta \boldsymbol{r}_{j}\left(t ; t_{0}\right)\right]\right\rangle_{t_{0}},
$$

where $\Delta \boldsymbol{r}_{j}\left(t ; t_{0}\right)=\boldsymbol{r}_{j}\left(t+t_{0}\right)-\boldsymbol{r}_{j}\left(t_{0}\right)$ is the displacement vector of the $j$ th particle in time $t$ and $\langle\cdots\rangle_{t_{0}}$ represents an average over initial times $t_{0}$. It is noted that $F_{s}(q, t)$ is one of the standard quantities in the studies of dynamic properties of supercooled liquids and glasses $[17,18]$. In Fig. 2, we plotted the decay profiles of $F_{s}(q, t)$ at a dimensionless wave number $\tilde{q}=2 \pi$ for $\tilde{n}=0.94,1.06,1.14$, and 1.21 . We see in Fig. 2 that the relaxation of $F_{s}(q, t)$ at $\tilde{n}=0.94$ can be expressed by a simple exponential function. Beyond the density $\tilde{n}=1.06$, however, $F_{s}(q, t)$ exhibits a two step, that is, fast $\beta$ and slow $\alpha$, relaxation, which is often mentioned as a characteristic sign of the slow relaxation in glass forming liquids. At the highest density $\tilde{n}=1.21$, the $F_{s}(q, t)$ does not show any decaying behavior [19]. One can define the structural relaxation time $\tilde{\tau}$ by $F_{s}(\tilde{q}=2 \pi, \tilde{\tau})=e^{-1}$, and this $\tilde{\tau}$ is plotted as a function of $\tilde{n}$ in Fig. 3. We notice in Fig. 3 that the relaxation time $\tilde{\tau}$ shows a strong dependence on density, which can be expressed by the power law $\left(\tilde{n}_{g, M D}-\tilde{n}\right)^{-\gamma}$ with $\tilde{n}_{g, M D} \simeq 1.15$ and $\gamma \simeq 1.31$ (solid line in Fig. 3 ).

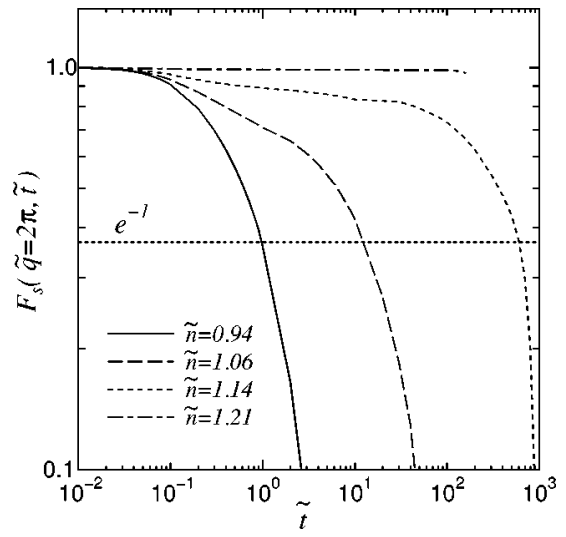

FIG. 2. Intermediate scattering function $F_{s}(\tilde{q}, \tilde{t})$ at a wave number $\tilde{q}=2 \pi$ for $\tilde{n}=0.94$ (solid line), 1.06 (dashed line), 1.14 (short dashed line), and 1.21 (dot-dashed line). The units of $q$ and $t$ are $\sigma^{-1}(\tilde{q}=q \sigma)$ and $\sqrt{m \sigma^{2} / k_{B} T}\left(\tilde{t}=t \sqrt{k_{B} T / m \sigma^{2}}\right)$, respectively.

We now consider energetics of the system based on the DFT and the configuration generated by the MD simulations. For a practical calculation of the DFT, we employ the Ramakrishnan and Yussouff (RY) free energy functional [4] because of its simplicity and physical clarity. The RY functional is given by

$$
F[n]=F_{i d}+F_{i n t}^{(2)}+F_{u n i},
$$

where

$$
F_{i d}=k_{B} T \int n(\boldsymbol{r}) \ln \left[\frac{n(\boldsymbol{r})}{n}\right] d \boldsymbol{r},
$$

$$
F_{\text {int }}^{(2)}=-\frac{1}{2} k_{B} T \iint[n(\boldsymbol{r})-n] C\left(\left|\boldsymbol{r}-\boldsymbol{r}^{\prime}\right|\right)\left[n\left(\boldsymbol{r}^{\prime}\right)-n\right] d \boldsymbol{r} d \boldsymbol{r}^{\prime} .
$$

Here, $F_{i d}$ and $F_{u n i}$ represent the ideal gas contribution and the excess free energy of the uniform liquid state $n(\boldsymbol{r})=n$, respectively. $F_{\text {int }}^{(2)}$ represents the second-order term in the expansion around the uniform liquid state, thus all terms higher

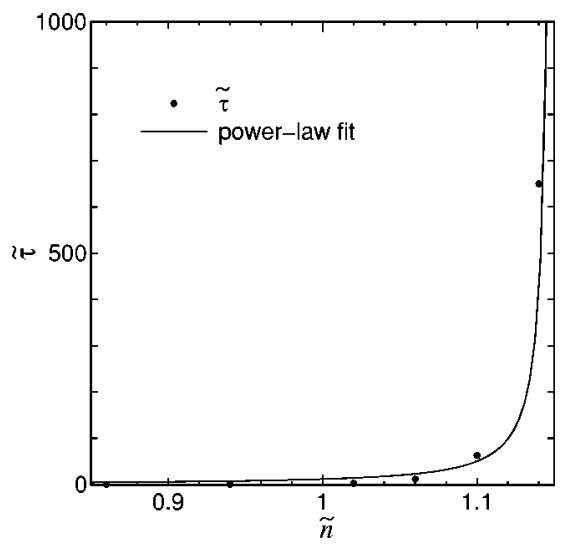

FIG. 3. Structural relaxation time $\tilde{\tau}$ as a function of density $\tilde{n}$ (closed circles). Solid line represents power-law fit $\left(\tilde{n}_{g, M D}-\tilde{n}\right)^{-\gamma}$ with $\tilde{n}_{g, M D}=1.15$ and $\gamma=1.31$. 
than second order are neglected. We note that $C(\boldsymbol{r})$ is the direct correlation function of the uniform liquid with density $n[20]$.

In order to evaluate the free energy of the system, we need the trial density field $n(\boldsymbol{r})$, for which we employ a conventional Gaussian superposition [6-10]; that is, the density field $n(\boldsymbol{r})$ is expressed by a sum of Gaussians with the centers located at $N$ sites $\left\{\boldsymbol{r}_{i}\right\}$, which are given by our MD simulation,

$$
n(\boldsymbol{r})=\sum_{i=1}^{N}\left(\frac{\alpha}{\pi}\right)^{3 / 2} \exp \left[-\alpha\left(\boldsymbol{r}-\boldsymbol{r}_{i}^{2}\right)\right] \equiv \sum_{i=1}^{N} z\left(\boldsymbol{r} ; \boldsymbol{r}_{\boldsymbol{i}}\right),
$$

where $\alpha^{-1}$, a variational parameter for the calculation of the free energy, is proportional to the mean square displacement of each particle. Small (large) $\alpha$ represents the uniform liquid (localized amorphous) state.

When $\alpha$ is very large, $F_{i d}$ is asymptotically represented by [6]

$$
F_{i d}(\alpha) \sim N k_{B} T\left[\left\{\frac{3}{2} \ln \left(\frac{\alpha}{\pi}\right)-\frac{3}{2}\right\}-\ln n\right] .
$$

For a small $\alpha$ region, we calculated the integral Eq. (5) numerically. We confirmed that $F_{i d}$ approaches zero when $\tilde{\alpha}$ $\rightarrow 0$ and noticed that $F_{i d}$ coincides with the value of Eq. (8) for $\tilde{\alpha} \gtrsim 20$.

It is easy to see that the interaction term $F_{\text {int }}$ can be divided into three parts as [6]

$$
F_{i n t}^{(2)}(\alpha)=-\frac{1}{2} N k_{B} T\left\{F_{i n t, 1}(\alpha)+F_{i n t, 2}(\alpha)-n \int C(r) d \boldsymbol{r}\right\},
$$

where $F_{\text {int }, 1}(\alpha)$ represents the self-interaction of a single Gaussian,

$$
F_{i n t, 1}(\alpha)=\iint z(\boldsymbol{r} ; \mathbf{0}) C\left(\left|\boldsymbol{r}-\boldsymbol{r}^{\prime}\right|\right) z\left(\boldsymbol{r}^{\prime} ; \mathbf{0}\right) d \boldsymbol{r} d \boldsymbol{r}^{\prime},
$$

and $F_{\text {int }, 2}(\alpha)$ represents the interaction between the two distinct Gaussians,

$$
\begin{aligned}
F_{i n t, 2}(\alpha)= & n \int g\left(r_{1}\right) d \boldsymbol{r}_{1} \iint z(\boldsymbol{r} ; \mathbf{0}) C\left(\left|\boldsymbol{r}-\boldsymbol{r}^{\prime}\right|\right) \\
& \times z\left(\boldsymbol{r}^{\prime} ; \boldsymbol{r}_{1}\right) d \boldsymbol{r} d \boldsymbol{r}^{\prime} .
\end{aligned}
$$

The pair distribution function $g(r)$ in this equation is determined from the MD simulation. With respect to the direct correlation function $C(r)$, we use Henderson-Grundke expression for $C(r)$, which is known to be reliable, though empirical, even for high-density hard sphere liquids [21].

The total free energy per particle relative to uniform state,

$$
\Delta f(\alpha)=\frac{F_{i d}(\alpha)+F_{i n t}^{(2)}(\alpha)}{N k_{B} T},
$$

is calculated as a function of the localization parameter $\tilde{\alpha}$ (see Fig. 4). It is seen in Fig. 4 that the free energy local
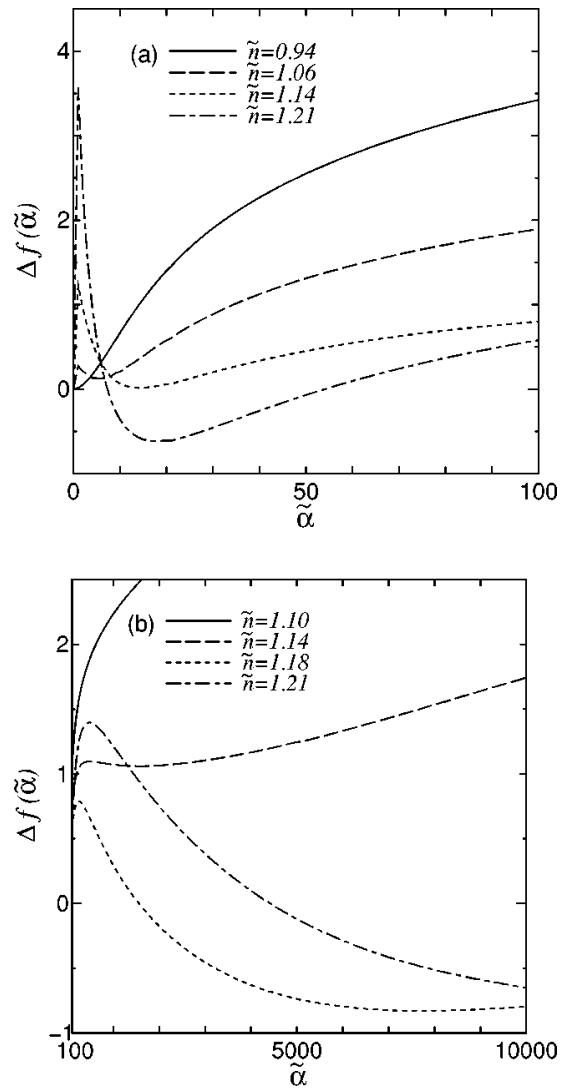

FIG. 4. Total free energy per particle relative to uniform liquid $\Delta f(\tilde{\alpha})$ as a function of localization parameter $\tilde{\alpha}$ for $\tilde{\alpha} \leqslant 100$ (a) and $\tilde{\alpha} \geqslant 100$ (b). The unit of $\tilde{\alpha}$ is $\sigma^{-2}\left(\tilde{\alpha}=\alpha \sigma^{2}\right)$.

minimum at finite $\alpha$, which represents an amorphous state, appears as the density is increased. As mentioned in Ref. [6], the local minimum appears as the result of the competition between the ideal gas (simple increasing function of $\alpha$ ) and the interaction terms. Figures 4 show that two local minima are located at $\tilde{\alpha} \simeq 13$ and 1600 for $\tilde{n}=1.14$. Das and Kaur also observed two local minima of $\Delta f(\alpha)$, which are called the weakly localized state for small $\alpha$ and the highly localized state for large $\alpha$ [9]. In addition, similar values for $\alpha$ have been reported in Refs. [6,9], which also use the RY functional, in relation to the local minimum of $\Delta f(\alpha)$. As stated in Ref. [9], the qualitative adequacy is ambiguous for the highly localized state with very high value of $\alpha$ since the RY form includes a perturbative expansion around the uniform state. In fact, based on the MD data, we estimated $\alpha$ for high-density states by relating it to the plateau value of the time-dependent mean square displacement of each particle, yielding $\tilde{\alpha} \simeq 50$ for $\tilde{n}=1.14$, for instance. From this, it is seen that the RY form does not give the proper estimation of the degree of localization compared with the results obtained in earlier works $[8,10]$.

In Fig. 5, we plotted the free energy differences $\Delta f$ of the weakly and highly localized states as a function of density $\tilde{n}$. From Fig. 5, we notice that the weakly localized state appears for $\tilde{n} \geqslant 1.06$ and the highly localized state appears for $\tilde{n} \geqslant 1.14$. For higher densities $\tilde{n} \gtrsim 1.15$, it is seen that the 


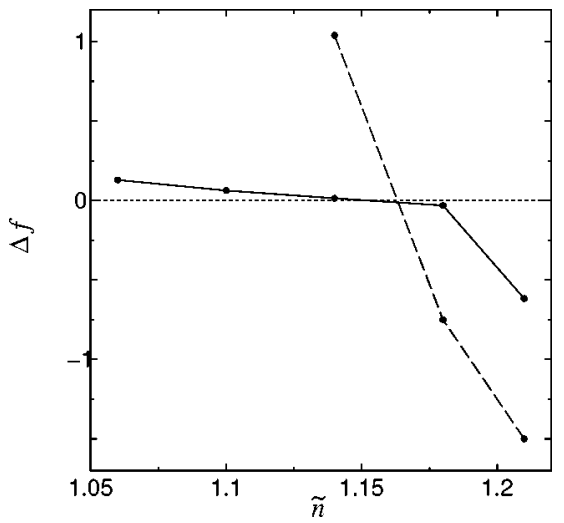

FIG. 5. Free energy differences $\Delta f$ of the weakly localized state (solid line) and the highly localized state (dashed line) as a function of density $\tilde{n}$.

highly localized state is more stable than the weakly localized state. Moreover, it is found in Fig. 5 that both weakly and highly localized states become more stable than the uniform state at around $\tilde{n}_{g, D F T} \simeq 1.15$, which is the liquid-glass transition density from the energetics based on the DFT. In passing, we note that our (random first order) glass transition density $\tilde{n}_{g, D F T}=1.15$ is rather close to that $\tilde{n}_{g}=1.14$, found in Ref. [6].

Finally, we compare our results from the energetics above with dynamic information supplied by our MD simulations. We find in Fig. 2 that the intermediate scattering function $F_{s}(q, t)$ begins to exhibit the two-step relaxation at density $\tilde{n} \simeq 1.06$, which corresponds precisely to the density where the free energy local minimum begins to appear in our DFT (see Figs. 4). Turning to the relaxation time $\tilde{\tau}$, we recall that the density dependence of $\tilde{\tau}$ could be described by the power law $\left(\tilde{n}_{g, M D}-\tilde{n}\right)^{-\gamma}$ with $\tilde{n}_{g, M D} \simeq 1.15$. This density happened to coincide with the density $n_{g, D F T}$, beyond which the localized state is more stable than the uniform liquid in the present DFT. From these results, we consider that the DFT based energetics and dynamic behaviors related to slow dynamics are well correlated with each other.
In this paper, we reconsidered the DFT approach to the glass transition in the hard sphere system, which was first undertaken by Singh et al. We obtained hard sphere glasses by MD simulations without recourse to the Bennett algorithm, and the information on particle configurations produced by the MD simulations is used as input data when the free energy is calculated based on the DFT. While only the uniform liquid state is stable at low density, the free energy local minimum begins to appear at high density $\tilde{n} \simeq 1.06$, where our MD shows that two-step relaxation begins to appear. This metastable glassy state becomes stable relative to the uniform liquid at $\tilde{n}_{g, M D}=1.15$. Slow relaxation, as represented by $F_{s}(q, t)$, turned out to be consistent with the energetics based on the DFT.

Before concluding this paper, we comment on recent developments in the studies of the DFT. In recent years, the so-called weighted density approximation (WDA) for the free energy functional has been developed [22,23] and the modified version has also been introduced [24]. Moreover, the fundamental measure theory (FMT) has been proposed [25] and is gathering considerable interest. It is well known that, for highly localized states, such methods are more accurate than the RY functional. This is because the former employs a nonperturbation approximation, whereas the latter relies on a perturbation expansion around the uniform state. Several workers have already investigated the glass transition by using the modified WDA method and found that the metastable localized state is located at $\tilde{\alpha} \simeq 100[8,10]$, in accordance also with our MD results. Although the DFT based on the RY functional is still useful because of its physical clarity, generality, and simplicity, we think that in view of the recent achievements, it is meaningful to employ the WDA or FMT method in order to obtain improved results.

Furthermore, we expect that the present DFT approach will be applied to more complex systems. As a model of a glass forming liquid, the binary Lennard-Jones [17] or softcore system [18] has been investigated by large scale MD simulations. Our approach can be readily applied to such a system and would give new insights into the glass transition from both thermodynamic and dynamic points of view.
[1] J. Jäckle, Rep. Prog. Phys. 49, 171 (1986).

[2] M.D. Ediger, C.A. Angell, and S.R. Nagel, J. Phys. Chem. 100, 13200 (1996).

[3] For reviews, see A.D.J. Haymet, Annu. Rev. Phys. Chem. 38, 89 (1987); D.W. Oxtoby, in Liquid, Freezing, and the Glass Transition, edited by J.P. Hansen, D. Levesque, and J. ZinnJustin (Elsevier, New York, 1990).

[4] T.V. Ramakrishnan and M. Yussouff, Phys. Rev. B 19, 2775 (1979).

[5] A.D. Haymet and D.W. Oxtoby, J. Chem. Phys. 74, 2559 (1981); D.W. Oxtoby and A.D. Haymet, ibid. 76, 6262 (1982).

[6] Y. Singh, J.P. Stoessel, and P.G. Wolynes, Phys. Rev. Lett. 54, 1059 (1985).

[7] M. Baus and J.L. Colot, J. Phys. C 19, L135 (1986).

[8] H. Löwen, J. Phys.: Condens. Matter 2, 8477 (1990).
[9] C. Kaur and S.P. Das, Phys. Rev. Lett. 86, 2062 (2001)

[10] C. Kaur and S.P. Das, Phys. Rev. E 65, 026123 (2002).

[11] C.H. Bennett, J. Appl. Phys. 43, 2727 (1972).

[12] X. Xia and P.G. Wolynes, Proc. Natl. Acad. Sci. U.S.A. 97, 2990 (2000).

[13] B.D. Lubachevsky and F.H. Stillinger, J. Stat. Phys. 60, 561 (1990); B.D. Lubachevsky, F.H. Stillinger, and N. Pinson, ibid. 60, 501 (1991).

[14] For all physical quantities, the following reduced units are used: $\tilde{r}=r / \sigma, \tilde{t}=t \sqrt{k_{B} T / m \sigma^{2}}, \tilde{n}=n \sigma^{3}(n \equiv N / V), \quad \tilde{q}=q \sigma$, and $\tilde{\alpha}=\alpha \sigma^{2}$.

[15] B.J. Alder and T.E. Wainwright, J. Chem. Phys. 31, 459 (1959).

[16] M.P. Allen and D.J. Tildesley, Computer Simulations of Liq- 
uids (Clarendon Press, Oxford, 1987).

[17] W. Kob and H.C. Andersen, Phys. Rev. E 52, 4134 (1995).

[18] R. Yamamoto and A. Onuki, Phys. Rev. E 58, 3515 (1998).

[19] It is remarked here that due to frequent collisions under compressed situations, a long time simulation at high density is prohibitively difficult to perform.

[20] J.P. Hansen and I.R. McDonald, Theory of Simple Liquids
(Academic Press, London, 1986).

[21] D. Henderson and E.W. Grundke, J. Chem. Phys. 63, 601 (1975).

[22] P. Tarazona, Phys. Rev. A 31, 2672 (1985).

[23] W.A. Curtin and N.W. Ashcroft, Phys. Rev. A 32, 2909 (1985).

[24] A.R. Denton and N.W. Ashcroft, Phys. Rev. A 39, 4701 (1989).

[25] Y. Rosenfeld, Phys. Rev. Lett. 63, 980 (1989). 